\title{
Low Speed Motion Optimization of Space Manipulator Based on Gradient Projection Method
}

\author{
Junjie Peng ${ }^{\mathrm{a}}$, Qingxuan Jia, Gang Chen and Yong Liu \\ Beijing University of Posts and Telecommunications in Beijing, China
}

\begin{abstract}
Low-speed crawling phenomenon may occur when space manipulators run at a low speed, which may bring big problem for manipulation work. A kind of low-speed optimization algorithm based on gradient projection method is pro-posed in this paper. The designing of continuous balanced proportional factor can effectively reduce the quantitative differences between the homogeneous solution and the special solution, avoiding the joint velocities oscillations. The low-speed crawling problem can be effectively improved by appropriate increase of joint velocities. And the effectiveness and correctness of the optimization algorithm are confirmed by the simulation.
\end{abstract}

\section{Introduction}

Due to large reduction ratio and high transmission efficiency, the harmonic reducers have been widely used in joint driving module of space manipulators. However, there has been some inevitable nonlinear friction in its own device, which causes big problem to space manipulators' movement. The manipulator will be in a state of pulsation when the joint speed is lower than a critical value, resulting in unstable movement of space manipulator [1-2]. Such low speed creeping phenomenon can seriously affect the control accuracy of the space manipulator, and what's worse, it may cause permanent damage to the manipulator.

For low speed creeping problem, Hung V M [3] proposed an application of an adaptive neuro-fuzzy controller for compensating friction and disturbance effects on robot manipulators, which improved the joint control accuracy of space manipu lator at a low speed effectively. Kermani M R [4] studied the closed-loop stability of the manipulator system by using the single state friction model, and improved the joint tracking accuracy of the manipulator though friction compensation.

Gradient projection method has been used to solve the problem of kinematic optimization control of redundant manipulators for a long time, and the use of performance index function can achieve a variety of subprime tasks [5]. Moreover, the optimization proportion factors are always empirical constants or piecewise functions obtained after multiple trial calculations [6], which make the algorithm inefficient and not universal. What is worse, the improper selection of the proportional factor can cause the severe oscillation problem of the joint velocity.

Liu Y [7] proposed a new Gradient Projection Method, which can effectively regulate the magnitude difference of the homogeneous solution and the special solution, but when the gradient vector performance function tends to 0 , the scalar function will tend to infinity. For the problem of scalar factor tending to infinity, Sun Kui [8] proposed a redundant manipulator gradient projection algorithm based on continuous scaling factor, which effectively improved the oscillation problem of joint velocity. The equation proposed was as follows:

$$
\dot{\boldsymbol{\theta}}=\boldsymbol{J}^{+} \dot{\boldsymbol{x}}+k_{c}\left(\boldsymbol{I}-\boldsymbol{J}^{+} \boldsymbol{J}\right) \nabla \boldsymbol{H}
$$

Where, the continuous scaling factor proposed by Sun is defined as $k_{c}=\lambda\left\|\boldsymbol{J}^{+} \dot{\boldsymbol{x}}\right\| /\left(\left\|\boldsymbol{J}^{+} \dot{\boldsymbol{x}}\right\|+\left\|\left(\boldsymbol{I}-\boldsymbol{J}^{+} \boldsymbol{J}\right) \nabla \boldsymbol{H}\right\|\right)$. What the problem is, it cannot guarantee the continuity of joint velocity.

For the problem above, this paper considering from the trajectory optimization level, proposes an improved gradient projection algorithm to solve the low-speed crawling problem.

\section{Mathematical Model of Space Manipulator}

\subsection{Kinematic Model}

As shown in Figure 1, the system considered in this paper consists of a base and a revolute-jointed manipulator (from link 1 to link $n$ ) which has $n$ degrees of freedom. It is assumed that the components of the system are all rigid bodies.

The symbols in Figure 1 are defined as follows:

$\Sigma_{I}$ : inertial coordinate system, which is the reference coordinate system of all recursive calculations;

$\Sigma_{b}$ : coordinate system of the base;

\footnotetext{
${ }^{a}$ Corresponding author: roger2010@bupt.edu.cn
} 
$\Sigma_{k}$ : coordinate system of link $k(k=1, \cdots, n-1, n)$;

$J_{k}$ : joint $k$ of the manipulator, which is used to connect link $k$ with link $k+1$;

$\boldsymbol{l}_{k-1, k}:$ vector from $\Sigma_{k-1}$ to $\Sigma_{k}$.

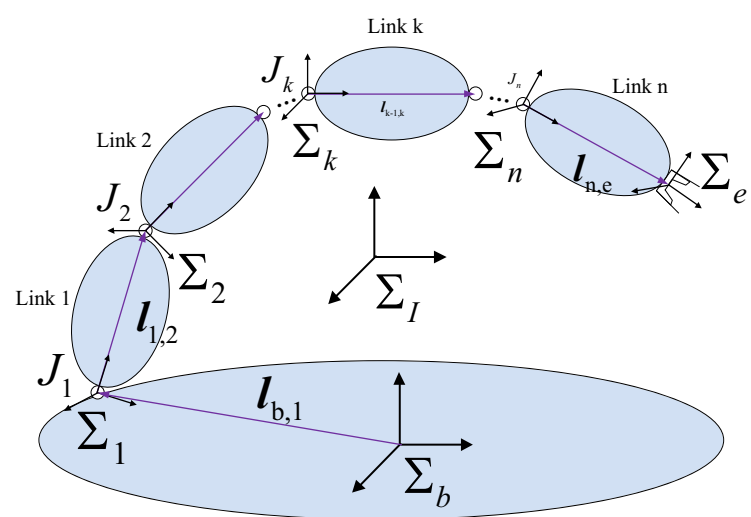

Figure 1. Simplified model of space manipulators

Define $\boldsymbol{x}_{e} \in \boldsymbol{R}^{m \times 1}$ as pose vector of the end-effector with respect to $\Sigma_{I} ; \boldsymbol{\theta} \in \boldsymbol{R}^{n \times 1}$ is defined as the joint angle. According to reference [9], then the general kinematics equation of space manipulators can be written as:

$$
\dot{\boldsymbol{x}}_{e}=\boldsymbol{J}_{b} \dot{\boldsymbol{x}}_{b}+\boldsymbol{J}_{m} \dot{\boldsymbol{\theta}}
$$

Where $\dot{\boldsymbol{x}}_{e}=\left[\boldsymbol{v}_{e}^{\mathrm{T}}, \boldsymbol{\omega}_{e}^{\mathrm{T}}\right]^{\mathrm{T}} \in \boldsymbol{R}^{6 \times 1}$ is velocity vector of the end-effector with respect to $\Sigma_{I}, \dot{\boldsymbol{x}}_{b}=\left[\boldsymbol{v}_{b}^{T}, \boldsymbol{\omega}_{b}^{T}\right]^{T} \in \boldsymbol{R}^{6 \times 1}$ is velocity vector of the base with respect to $\Sigma_{I}$, $\dot{\boldsymbol{\theta}}=\left[\dot{\theta}_{1}, \dot{\theta}_{2}, \cdots, \dot{\theta}_{n}\right]^{\mathrm{T}} \in \boldsymbol{R}^{n \times 1}$ is joint angular velocity vector, $\boldsymbol{J}_{b} \in \boldsymbol{R}^{6 \times 6}$ is Jacobian matrix denoting the relationship between velocity of the base and velocity of the endeffector, $\boldsymbol{J}_{m} \in \boldsymbol{R}^{6 \times 6}$ is Jacobian matrix denoting the relationship between joint angular velocity and velocity of the end-effector.

It is assumed that the initial linear and angular momentum are equal to 0 , and no external forces or torques act on the whole system in the free-floating mode. According to conservation of momentum and angular momentum, we can easily obtain that:

$$
\boldsymbol{H}_{b} \dot{\boldsymbol{x}}_{b}+\boldsymbol{H}_{b m} \dot{\boldsymbol{\theta}}=\boldsymbol{0}
$$

Where $\boldsymbol{H}_{b}$ and $\boldsymbol{H}_{b m}$ denote inertia matrix of base and coupled inertia matrix respectively. Substitute Eq. (4) into Eq. (3) leads to the relationship as follows:

$$
\begin{aligned}
& \dot{\boldsymbol{x}}_{b}=\boldsymbol{J}_{\text {bm }} \dot{\boldsymbol{\theta}} \\
& \dot{\boldsymbol{x}}_{e}=\boldsymbol{J}_{\text {float }} \dot{\boldsymbol{\theta}}
\end{aligned}
$$

Where $\quad \boldsymbol{J}_{\text {float }}=\boldsymbol{J}_{m}-\boldsymbol{J}_{b} \boldsymbol{H}_{b}^{-1} \boldsymbol{H}_{b m} \quad$ denotes the generalized Jacobian matrix of space manipulators; $\boldsymbol{J}_{b m}=-\boldsymbol{H}_{b}^{-1} \boldsymbol{H}_{b m}$ denotes the relationship between joint angular velocity and velocity of the base.

The general solution of Eq. (3) is as follows:

$$
\dot{\boldsymbol{\theta}}=\boldsymbol{J}_{\text {float }}^{+} \dot{\boldsymbol{x}}_{\boldsymbol{e}}+k\left(\boldsymbol{I}-\boldsymbol{J}_{\text {float }}^{+} \boldsymbol{J}_{\text {float }}\right) \nabla \boldsymbol{H}
$$

Where $\boldsymbol{J}_{\text {float }}^{+}$is defined as the pseudo inverse matrix of the Jacobian matrix; and $\boldsymbol{I} \in \boldsymbol{R}^{6 \times 6}$ is defined as the unit matrix; $k$ is the optimal proportion factor; and $\boldsymbol{\nabla H}$ is the gradient vector of performance index function.

\subsection{Dynamic Model}

In this paper, the joint friction of the space manipulator is described by the Lugre model, the expressions of which are as follows:

$$
\begin{aligned}
& \frac{d z}{d t}=\dot{\theta}-\frac{|\dot{\theta}|}{\boldsymbol{g}(\dot{\theta})} z \\
& \boldsymbol{T}_{f}=\sigma_{0} z+\sigma_{1} \frac{d z}{d t}+\sigma_{2} \dot{\theta} \\
& \boldsymbol{g}(\dot{\theta})=\frac{1}{\sigma_{0}}\left[\boldsymbol{T}_{c}+\left(\boldsymbol{T}_{s}-\boldsymbol{T}_{c}\right) e^{-\left(\dot{\theta} / \dot{\theta}_{e}\right)^{2}}\right]
\end{aligned}
$$

Where the parameters are defined as follows:

$z$ : Microscopic average bristle deflection;

$\boldsymbol{T}_{f}:$ Joint friction torque;

$\boldsymbol{T}_{\boldsymbol{c}}$ : Coulomb friction torque;

$\boldsymbol{T}_{\boldsymbol{s}}:$ Stiction friction torque;

$\sigma_{0}:$ Bristle stiffness coefficient;

$\sigma_{1}$ : Damping coefficient;

$\sigma_{2}:$ Viscous friction coefficient;

$\dot{\theta}_{e}:$ Stribeck velocity, which is obtained from $d z / d t=0$.

According to reference [10], when joint angular velocity comes lower than the Stribeck velocity, $\boldsymbol{z}$ would be a rand vector within a certain range, which will lead to the low speed creeping problem.

Take the general dynamic model for reference [11], and the dynamic equation of space manipulator considering the joint friction can be established as follows:

$$
\boldsymbol{M}(\theta) \ddot{\theta}+\boldsymbol{C}(\theta, \dot{\theta})+\boldsymbol{T}_{f}=\boldsymbol{\tau}
$$

Where $\boldsymbol{M}(\theta)$ is the Inertia matrix; $\ddot{\theta}$ is the joint acceleration; $\boldsymbol{C}(\theta, \dot{\theta})$ is the nonlinear term contains Coriolis and centrifugal torques; $\boldsymbol{\tau}$ is the joint driving torque; while $\boldsymbol{T}_{f}$ is the Lugre friction torque.

\section{Low Speed Motion Optimization Algorithm}

The inverse kinematics solution of space manipulator can be rewritten as:

$$
\dot{\boldsymbol{\theta}}=\boldsymbol{J}_{\text {float }}^{+} \dot{\boldsymbol{x}}_{e}+k_{m}\left(\boldsymbol{I}-\boldsymbol{J}_{\text {float }}^{+} \boldsymbol{J}_{\text {float }}\right) \nabla \boldsymbol{H}
$$



as:

The minimum performance index function is defined

$$
\boldsymbol{H}=\sum_{i=1}^{n} \frac{1}{\left(\dot{\theta}_{\text {now }}^{2}(i)-\dot{\theta}_{\text {limit }}^{2}(i)\right)^{2}}
$$

Where $\dot{\theta}_{\text {now }}(i)$ is the joint angular velocity of joint $i$, $\dot{\theta}_{\text {limit }}$ is the joint critical velocity for low speed crawling, which can also be called Stribeck velocity.

Take differential of the Eq.(9), we can obtain:

$$
\nabla \boldsymbol{H}=\left\{\frac{\partial \boldsymbol{H}}{\partial \theta_{1}}, \frac{\partial \boldsymbol{H}}{\partial \theta_{2}}, \cdots, \frac{\partial \boldsymbol{H}}{\partial \theta_{n}}\right\}
$$

According to reference [12], define the motion optimization parameter as $\gamma=\left.\frac{d \boldsymbol{H}}{d t}\right|_{\min }$, and only when $\gamma<0$ can $\boldsymbol{H}$ be optimized. So that as the amplification factor will be set as $\lambda=0$ when $\gamma \geq 0$.

As is clear that the scale factor would lead to quantitative problem in the gradient projection equation, the continuous balanced proportional factor is proposed in this paper as:

$$
k_{m}=\lambda \frac{\left\|\boldsymbol{J}^{+} \dot{\boldsymbol{X}}\right\|}{\left\|\boldsymbol{J}^{+} \dot{\boldsymbol{X}}+\left(\boldsymbol{I}-\boldsymbol{J}^{+} \boldsymbol{J}\right) \nabla \boldsymbol{H}\right\|+\rho}
$$

Where $\rho$ is a constant rational factor, which can guarantee that the continuous balanced proportional factor $k_{m}$ won't be infinite.

In addition, considering that the joint angular velocity at initial and end time is inevitable to be quite small, we just do the optimization from $t_{1}$ to $t_{2}$.

\section{Numerical Simulations}

\subsection{Establishment of Simulation Model}

A seven link space manipulator mounted on a base is considered with parameters $d_{1}=d_{7}=1.2 \mathrm{~m} ; d_{4}=0.52 \mathrm{~m}$; $d_{2}=d_{3}=d_{5}=d_{6}=0.53 \mathrm{~m} ; a_{3}=a_{4}=5.8 \mathrm{~m}$. And the joint frames based on DH method are shown in Figure 2, the pose of $\Sigma_{0}$ is $\left[-0.1 \mathrm{~m}, 0 \mathrm{~m}, 1.5 \mathrm{~m}, 0^{\circ}, 0^{\circ}, 0^{\circ}\right]$. The relative parameters are shown in Table 1.

Table 1 DH parameters of 7-DOF space manipulator

\begin{tabular}{|c|c|c|c|c|c|c|c|c|c|}
\hline \multicolumn{2}{|c|}{ Parameters } & Base & Link 1 & Link 2 & Link 3 & Link 4 & Link 5 & Link 6 & Link 7 \\
\hline \multicolumn{2}{|c|}{ Mass/(kg) } & $10^{5}$ & 30 & 30 & 70 & 75 & 30 & 30 & 40 \\
\hline \multirow{3}{*}{\multicolumn{2}{|c|}{${ }^{i} \boldsymbol{p}_{i} /(\mathrm{m})$}} & 0 & 0 & -0.265 & 2.9 & 2.7 & 0 & 0 & 0 \\
\hline & & 0 & -0.265 & 0 & 0 & 0 & 0 & 0 & 0 \\
\hline & & 0 & 0 & 0 & 0 & 0.5 & 0.265 & 0.265 & 0.6 \\
\hline \multirow{6}{*}{$\begin{array}{l}I_{k} / \\
(\mathrm{kg} . \\
\left.\mathrm{m}^{2}\right)\end{array}$} & $I_{x x}$ & $10^{5}$ & 0.98 & 0.57 & 1.32 & 1.91 & 0.98 & 0.98 & 5.18 \\
\hline & $I_{y y}$ & $10^{5}$ & 0.57 & 0.98 & 197.2 & 243.4 & 0.98 & 0.98 & 5.18 \\
\hline & $I_{z z}$ & $10^{5}$ & 0.98 & 0.98 & 197.2 & 242.9 & 0.57 & 0.57 & 0.75 \\
\hline & $I_{x y}$ & 0 & 0 & 0 & 0 & 0 & 0 & 0 & 0 \\
\hline & $I_{y z}$ & 0 & 0 & 0 & 0 & 0 & 0 & 0 & 0 \\
\hline & $I_{z x}$ & 0 & 0 & 0 & 0 & -4 & 0 & 0 & 0 \\
\hline
\end{tabular}

\begin{tabular}{|c|c|c|c|c|}
\hline Link & $\alpha$ & $a$ & $\theta$ & $d$ \\
\hline 1 & 0 & 0 & 0 & $d_{1}$ \\
\hline 2 & $\pi / 2$ & 0 & $\pi / 2$ & $d_{2}$ \\
\hline 3 & $-\pi / 2$ & 0 & 0 & $d_{3}+d_{4}+d_{5}$ \\
\hline 4 & 0 & $a_{3}$ & 0 & 0 \\
\hline 5 & 0 & $a_{4}$ & 0 & 0 \\
\hline 6 & $\pi / 2$ & 0 & $\pi / 2$ & $d_{6}$ \\
\hline 7 & $\pi / 2$ & 0 & 0 & $d_{7}$ \\
\hline
\end{tabular}

Table 2 Kinetic parameters of 7-DOF space manipulator

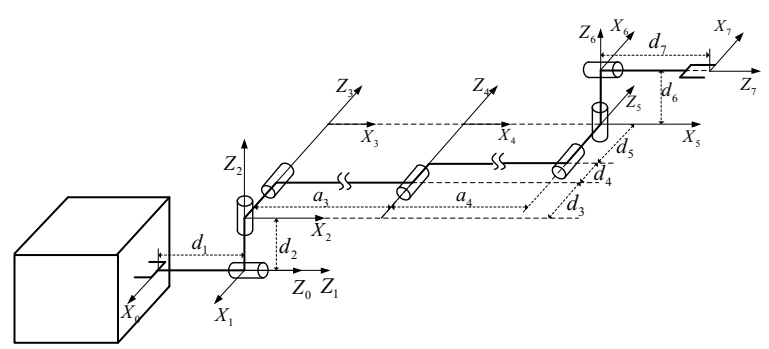

Figure 2. 7-DOF space manipulator

\subsection{Simulation Parameter Settings}

The initial joint angles: $\boldsymbol{\theta}_{i n i}=\left[-50^{\circ},-170^{\circ}, 150^{\circ},-60^{\circ}\right.$, $\left.130^{\circ}, 170^{\circ}, 0\right]$;

The desired pose of end effector: $\boldsymbol{x}_{e}=[9.5,0,3.0$, $0,-0.5,-2.0]$;

The simulation time: $t_{i n i}=0, t_{\text {final }}=40 \mathrm{~s}, t_{0}=0.1 \mathrm{~s}$, $t_{1}=3 \mathrm{~s}, t_{2}=37 \mathrm{~s}$; 
According to reference [13], the Stribeck velocity can be set as: $\left|\dot{\theta}_{\text {limit }}\right|=0.2^{\circ} / \mathrm{s}$, and the constant rational factor is $\rho=0.0001$.

\subsection{Simulation Results and Analysis}

Simulations are performed to examine the effectiveness of optimization algorithm. The joint angular velocity and joint torque of space manipulator before optimization are as follows:

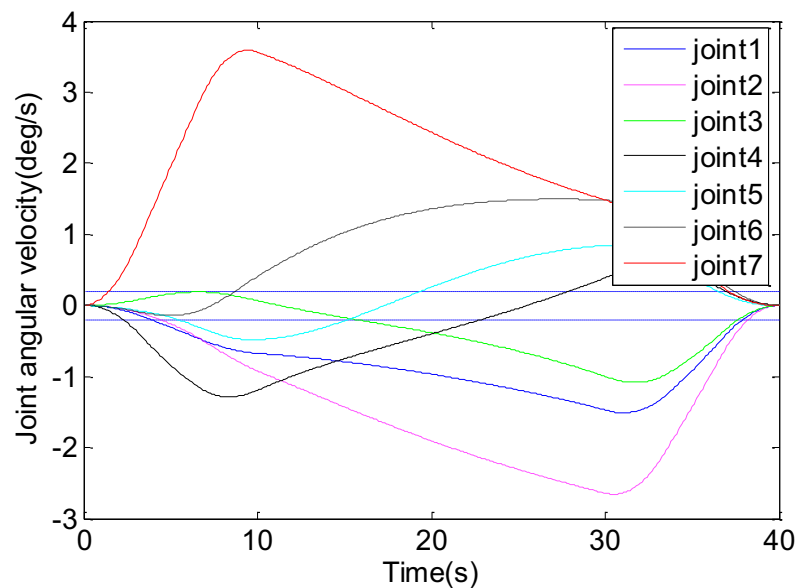

a. joint angular velocity

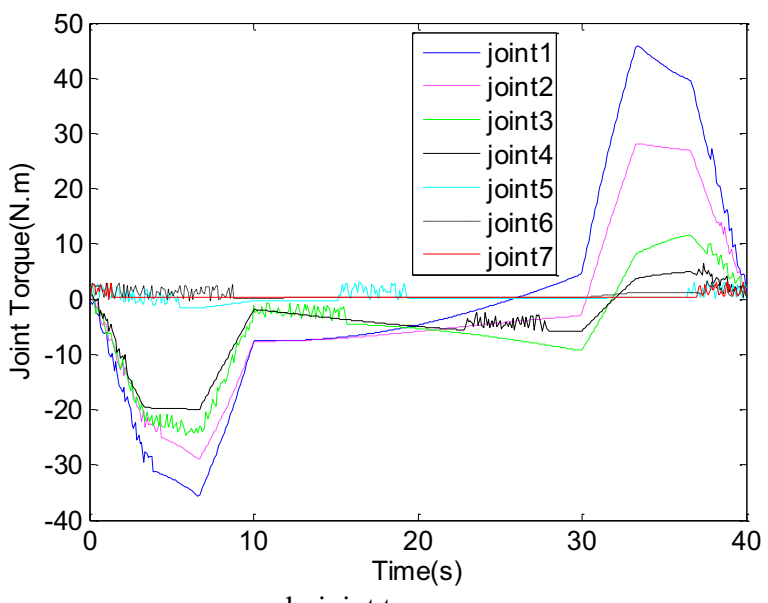

b. joint torque

Figure 3. The motion results of space manipulator before optimization

As can be seen from Figure 3, the joint angular velocity of space manipulator is less than the critical velocity in a long time, which leads to nonlinear feature of the corresponding joint friction, thus it causes the joint torque jittering.

The joint angular velocity and joint torque of space manipulator after optimization are as follows:

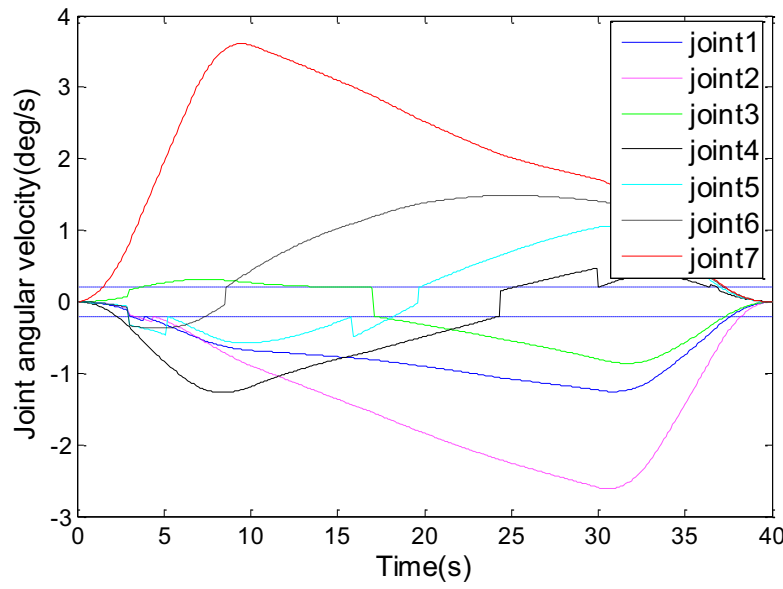

a. joint angular velocity

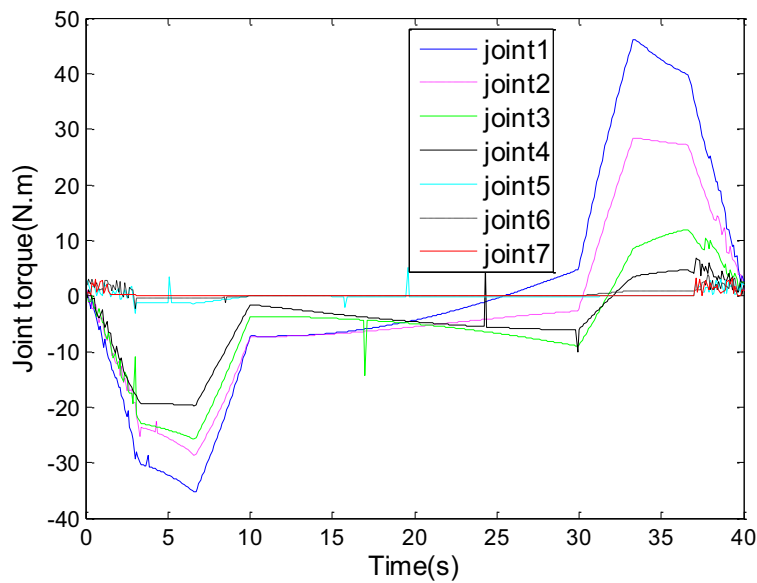

b. joint torque

Figure 4. The motion results of space manipulator after optimization

As can be seen from Figure 4, the joint angular velocity of space manipulator has been optimized during $3 \mathrm{~s}$ and $37 \mathrm{~s}$. And after optimization, the time in which the joint angular velocity is less than the critical velocity can be much less. The joint torque's jittering can be reduced largely though the joint torque would express a sudden increase because of the joint velocity's rapid increase, which shows that the low speed crawling problem of space manipulator can be improved effectively by using the low speed motion optimization algorithm proposed in this paper.

\section{Conclusions}

The low speed creeping problem of space manipulator has been improved in this paper by using the improved gradient projection method, which effectively guarantees that the joint velocity increase to a certain value larger than the Stribeck velocity. The correctness and effectiveness of the proposed algorithm are verified by numerical simulations.

\section{ACKNOWLEDGEMENT}


This project is supported by the National Natural Science Foundation of China (61573066) and the National Natural Science Foundation of China (61403038).

\section{References}

1. Y. Wang, Z. Xiong, H. Ding. Friction compensation design based on state observer and adaptive law for high-accuracy positioning system. Progress in natural science, 16(2): 147-152(2006)

2. J. Rong, Y Yang, J Li, C. Hu, B. Liu. Research on modeling and control scheme of space manipulator. Journal of Astronautics, 33(11): 1564-1569(2012)

3. V. M. Hung, H. J. Na. Adaptive neural fuzzy control for robot manipulator friction and disturbance compensator. Control, Automation and Systems, (2008)

4. M. R. Kermani, M. Wong, R. V. Patel. Friction compensation in low and high-reversal-velocity manipulators. Robotics and Automation, (2004)

5. M. S. Tisius. An empirical approach to performance criteria and redun-dancy resolution. University of Texas, Austin(2004)

6. J. Chen, J. Liu. Avoidance of obstacles and joint limits for end-effector tracking in redundant manipulators. Control, Automation, Robotics and Vision, (2002)

7. Y. Liu, J. Zhao, B. Xie. Obstacle avoidance for redundant manipulators based on a Novel Gradient Projection Method with a functional scalar. Robotics and Biomimetics (ROBIO), 2010 IEEE International Conference on. IEEE, 2010: 1704-1709

8. K. Sun, Z. Xie, J. Hu. Gradient projection method of kinematic redundant manipulator based on continuous scale factor. Journal of Jilin Uni-versity (Engineering and Technology Edition), 39(5), (2009)

9. Y. Xu, H. Y. Shum. Dynamic control and coupling of a free-flying space robot system. Journal of Robotic Systems, 11(7): 573-589(1994)

10. C. C. De Wit, H. Olsson, K. J. Astrom. A new model for control of systems with friction. Automatic Control, IEEE Transactions on, 40(3): 419-425(1995)

11. S. Abiko, G. Hirzinger. Adaptive control for a torque controlled free-floating space robot with kinematic and dynamic model uncertainty. Intelligent Robots and Systems, (2009)

12. L. Ti, Q. Zhang, Z. Yang. Control of redundant robots based on the motion optimizability measure. Robotics and Automation, (1994)

13. C. C. De Wit, P. Lischinsky. Adaptive friction compensation with par-tially known dynamic friction model. International journal of adaptive control and signal processing, 11(1): 65-80(1997) 